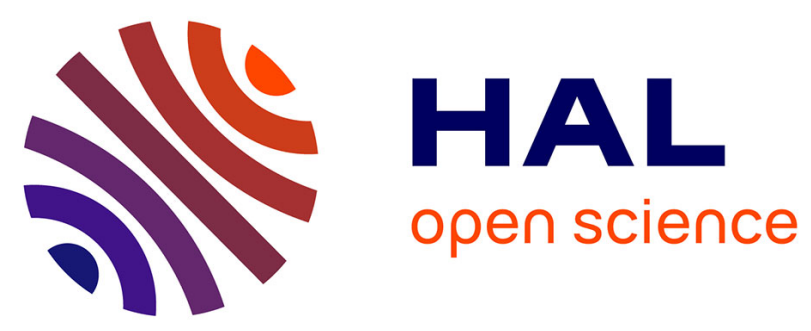

\title{
Interaction with 2,4-dinitrophenol correlates with polyreactivity, self-binding, and stability of clinical-stage therapeutic antibodies
}

Dimitrov Jordan, Maxime Lecerf, Stephanie Depinay, Rémi Noé, Jordan D Dimitrov

\section{To cite this version:}

Dimitrov Jordan, Maxime Lecerf, Stephanie Depinay, Rémi Noé, Jordan D Dimitrov. Interaction with 2,4-dinitrophenol correlates with polyreactivity, self-binding, and stability of clinical-stage therapeutic antibodies. Molecular Immunology, 2021, 140, pp.233-239. 10.1016/j.molimm.2021.10.019 . hal03547746

\section{HAL Id: hal-03547746 \\ https://hal.sorbonne-universite.fr/hal-03547746}

Submitted on 28 Jan 2022

HAL is a multi-disciplinary open access archive for the deposit and dissemination of scientific research documents, whether they are published or not. The documents may come from teaching and research institutions in France or abroad, or from public or private research centers.
L'archive ouverte pluridisciplinaire HAL, est destinée au dépôt et à la diffusion de documents scientifiques de niveau recherche, publiés ou non, émanant des établissements d'enseignement et de recherche français ou étrangers, des laboratoires publics ou privés. 
Interaction with 2,4-dinitrophenol correlates with polyreactivity, self-binding, and stability of human therapeutic antibodies

\begin{abstract}
Valentin Dietlin-Auril ${ }^{1, *}$, Maxime Lecerf ${ }^{1, *}$, Stephanie Depinay ${ }^{1}$, Rémi Noé and Jordan D. Dimitrov $^{1}$
\end{abstract}
${ }^{1}$ Centre de Recherche des Cordeliers, INSERM, Sorbonne Université, Université de Paris, 75006 Paris, France

${ }^{*}$ These authors contributed equally to the study

Contact address:

Jordan D. Dimitrov

Centre de Recherche des Cordeliers

15, rue de l'Ecole de Médecine

75006 Paris - France

E-mail: jordan.dimitrov@sorbonne-universite.fr / jordan.dimitrov@inserm.fr

Tel: +33144278206 


\begin{abstract}
Therapeutic antibodies should cover particular physicochemical and functional requirements for successful entry into clinical practice. Numerous experimental and computational approaches have been developed for early identification of different unfavourable features of antibodies. Immune repertoires of healthy humans contain a fraction of antibodies that recognize nitroarenes. These antibodies have been demonstrated to manifest antigen-binding polyreactivity. Here we observed that $>20 \%$ of 112 clinical stage therapeutic antibodies show pronounced binding to 2,4-dinitrophenol conjugated to albumin. This interaction predicts a number of unfavourable functional and physicochemical features of antibodies such as polyreactivity, tendency for self-association, stability and expression yields. Based on these findings we proposed a simple approach that may add to the armamentarium of assays for early identification of developability liabilities of antibodies intended for therapeutic use.
\end{abstract}




\section{Introduction}

Therapeutic monoclonal antibodies (mAbs) have been efficiently used for treatment of wide range of pathological conditions, including cancer, autoimmunity, infection- and neurological diseases. Currently there are more than 80 clinically approved therapeutic mAbs; hundreds other molecules are in clinical trials or in development (Kaplon and Reichert, 2021). Besides binding to its biological target, the therapeutic mAbs needs to fulfil certain additional criteria for successfully reaching clinical stage. Thus, a number of physicochemical or functional characteristics of the Ig molecule have been identified that can unfavourably influence production yields, solubility, storage stability, and pharmacokinetics (Jarasch et al., 2015; Steinmeyer and McCormick, 2008; Xu et al., 2019). These unfavourable developability features include low protein stability, vulnerability to chemical modifications, propensity for aggregation and self-association, as well as tendency for recognition of unrelated antigens i.e. polyreactivity. A variety of analytical methods and bioinformatics algorithms have been created and applied for detection of physicochemical or binding traits that can negatively impact the development of therapeutic mAb drug (Jain et al., 2017; Y. Liu et al., 2014; Raybould et al., 2019; Sharma et al., 2014; Xu et al., 2019; Y. Zhang et al., 2020). In their study Jain et al (Jain et al., 2017) undertook holistic approach and investigated the biophysical features and polyreactivity of 137 clinical-stage mAbs, using a set of 12 different assays for assessment of different problematic features. This study revealed important relationships between different physicochemical characteristics of mAbs of relevance for developability. Moreover, it showed that the number of developability issues progressively decreased with progression of mAb 
molecules through the development pipeline with no or only minimal number of issues for clinically approved mAbs.

Polyreactive Abs represent a significant component of the Ab repertoires of healthy individuals, representing 10-20\% of circulating Abs (Dimitrov et al., 2013). They play important physiological functions by participating in the defence against pathogens and in immune regulation. However, polyreactivity is undesirable feature of therapeutic mAbs candidates. Indeed, antigen-binding promiscuity was demonstrated to compromise the pharmacokinetics of mAbs (Avery et al., 2018; Hotzel et al., 2012). Due to their reactivity with endogenous antigen the polyreactive mAbs have considerably shorter half-life (Sigounas et al., 1994). Moreover, such mAbs may cause unwanted reactions due to off-target bindings. Several approaches have been employed for determining polyreactivity of therapeutic mAbs. Some of these approaches relay on immunosorbent assay (ELISA), where the reactivity of mAbs towards panels of distinct antigens or baculovirus particles is examined (Hotzel et al., 2012). Another approach (polyspecificity reagent assay) consists in interrogating binding properties of mAbs in solution using large repertoires of target antigens from eukaryotic cell lysates and yeast-displayed mAbs (Dostalek et al., 2017; Xu et al., 2013).

More than 40 years ago it was observed that a fraction of circulating Abs in virtually all healthy humans have a considerable reactivity for xenobiotic aromatic compound 2,4-dinitrophenol (DNP) (Farah, 1973; Karjalainen and Makela, 1976). Importantly it was also observed that the Abs recognizing DNP display antigen-binding polyreactivity (Berneman et al., 1993; Muhumuza et al., 1998; Ortega et al., 1984). Thus, this compound was successfully applied as a probe for purification of polyreactive Abs from pooled IgG preparations or for measuring of the changes 
in levels of polyreactive Abs in sera of patients with autoimmune disease (Bruley-Rosset et al., 2003; S. Gunti et al., 2015; S. Gunti and Notkins, 2015).

However to the best of our knowledge, the capacity of DNP to interrogate therapeutic mAbs for polyreactivity or other developability features was never tested. In the present study we took advantage of the comprehensive data provided in the study of Jain et al (Jain et al., 2017) and correlated the reactivity of 112 mAbs towards DNP with 12 different assays for assessment of physicochemical features and polyreactivity. Our data revealed that reactivity to DNP is a strong predictor of polyreactivity of mAbs. Notably, the binding of mAbs to DNP also correlated strongly and predicted other unwanted features such as: tendency for self-association, protein stability, reactivity to other IgG molecules, and expression yield. Based on these data we propose assessment of reactivity against DNP as a simple strategy for detection of polyreactivity and other developability issues of candidate therapeutic Abs.

\section{Materials and Methods}

\section{Recombinant IgG1 molecules}

We used 112 samples with variable region sequences corresponding to mAbs that are approved for clinical use or reached Phase II and Phase III clinical trials. A detailed information about the used repertoire of recombinant mAbs was provided in the article of Jain et al (Jain et al., 2017). All mAbs were expressed as IgG1 in HEK293 expression system and purified by protein G affinity chromatography. 


\section{Enzyme-linked immunosorbent assay}

96-well polystyrene plates NUNC MaxiSorp ${ }^{\text {TM }}$ (Thermo Fisher Scientific, Waltham, MA) were coated with Albumin, dinitrophenyl (Sigma-Aldrich St. Louis, MO, Ref \# A6661) here referred to as DNP-BSA or with unconjugated BSA. The protein were diluted in PBS to $10 \mu \mathrm{g} / \mathrm{ml}$ and incubated for 1 hour at room temperature with volume of $50 \mu \mathrm{l} /$ well. Next, plates were blocked by incubation for 1 hour at room temperature with PBS containing $0.25 \%$ Tween 20 with volume of $200 \mu \mathrm{l} /$ well. For assessment of the immunoreactivity toward DNP-BSA and BSA, all analyzed mAbs were diluted to $10 \mu \mathrm{g} / \mathrm{ml}$ in PBS containing $0.05 \%$ Tween 20 (PBS-T) and incubated for 1 hour at RT with immobilized targets in volume of $50 \mu \mathrm{l} /$ well. All plates included also a standard, which represented $100 \mu \mathrm{g} / \mathrm{ml}$ of pooled human IgG (IVIg, Endobulin, Baxter, USA). In alternative experimental setting, selected antibodies demonstrating the highest DNPBSA activity were analyses at serial ( $3 \times$ each step) dilutions starting from concentration of 30 $\mu \mathrm{g} / \mathrm{ml}(200 \mathrm{nM})$. Moreover, the reactivity of human pooled IgG preparation towards DNP-BSA was analyzed at serial $(2 \times$ each step) dilutions starting from concentration of $5 \mathrm{mg} / \mathrm{ml}(33.5$ $\mu \mathrm{M})$. After incubation with Abs, the plates were washed at least 5 times with PBS-T and then incubated for 1 hour at room temperature with anti-human IgG (Fc-specific, clone JDC-10, HRPconjugated, Southern Biotech, Birmingham, AL) diluted $3000 \times$ in PBS-T and incubated with volume of $50 \mu \mathrm{l} /$ well. Following 5 times washing with PBS-T, the reactivity of mAbs and IVIG was revealed by addition of $o$-phenylenediamine dihydrochloride (Sigma-Aldrich). The optical density at $492 \mathrm{~nm}$ was determined after stopping the reaction with $2 \mathrm{M} \mathrm{HCl}$ by using a microplate reader (Infinite 200 Pro, Tecan, Männedorf, Switzerland).

\section{Statistical analyses}


Amino acid sequences of variable regions of mAbs used in this work were retrieved from IMGT database (http://www.imgt.org/) and from sequence data provided along with the publication of Jain et al (Jain et al., 2017). The raw data from analyses of mAbs with 12 different developability assays were also obtained from information provided with the article of Jain et al. To adjust the reactivity of different mAbs to DNP-BSA measured on different plates we first divided the reactivity of each mAb to DNP-BSA by the one against BSA. Same procedure was performed for control of pooled human IgG, which was present at each plate at concentration of $100 \mu \mathrm{g} / \mathrm{ml}$. After the reactivity of mAbs was expressed as a fold of DNP-BSA binding in relation to the binding of the internal control pooled human IgG. The data of reactivity of mAbs for DNP-BSA were subjected to non-parametric Spearman correlation analyses using GraphPad Prism v.6 software (La Jolla, CA). The CDRs and framework regions of heavy and light chains Ig domains of mAbs were determined by using human germ-line genes as reference. The reactivity towards DNP-BSA was correlated with: (i) number of mutations in variable regions (data obtained using IMGT/DomainGapAlign tool), (ii) number of individual amino acid resides or their sums grouped by types (polar, acidic, basic, aromatic and hydrophobic) in each CDR or framework region, (iii) grand average of hydropathy (GRAVY) value in each CDR or framework region (data computed using GRAVY Calculator www.gray-calculator.de) and (iv) the isoelectric point (pl) value in each CDR or FR (data computed using the "IPC2.peptide.Conv2D" parameter of the Isoelectric Point Calculator 2.0 http://ipc2.mimuw.edu.pl). The reactivity of mAbs towards DNP was correlated with number of individual amino acid resides or their sums grouped by types (polar, acidic, basic, aromatic and hydrophobic) in all CDRs and framework regions of $V_{H}$ and $V_{L}$. The reactivity to DNP-BSA of mAbs was also correlated with the values 
from 12 different assays used in a previous report for assessment of physiochemical properties and polyreactivity of mAbs. A significant correlation was considered only if $\mathrm{P}$ value $\leq 0.05$.

\section{Results}

First, we studied the presence of anti-DNP in human immunoglobulin (Ig) repertoires. As a source of IgG we used intravenous Ig (IVIg), which represent therapeutic pooled human IgG isolated from plasmas of thousands of healthy blood donors. Thus, IVIg can be considered as representative of human Ig repertoires at population level. We immobilized on ELISA plates DNP conjugated to BSA (DNP-BSA) and assessed the reactivity of pooled IgG. The obtained data clearly show that human pooled IgG has a reactivity to DNP-BSA (Fig. 1A). This result is accordance with findings that reactivity against DNP is normally present in human immune repertoire (Ortega et al., 1984). The obtained result also confirmed the validity of using IVIG as a control in the subsequent experiments in this study. ED38 is a human polyreactive IgG1 widely applied as positive control in assays for detection of promiscuous binding of antibodies. We compared the binding of ED38 to DNP-BSA with binding of a monoreactive human IgG1 N07G05. The polyreactive ED38 demonstrated a strong reactivity for DNP-BSA, whereas the monoreactive IgG1 did not bind to DNP-BSA (Fig. 1B).

Next, to assess the prevalence of DNP-binding among therapeutic mAb molecules, we investigated a repertoire of $112 \mathrm{mAbs}$ with variable regions matching clinical-stage therapeutic molecules (kindly provided by Adimab, USA). The variable regions sequences of 44 molecules matched the variable regions of approved therapeutic mAbs whereas the rest 68 mAbs 
corresponded to molecules that are currently in phase 2 or 3 clinical trials. All mAbs were expressed as human $\operatorname{IgG1}$ and their physicochemical and binding properties were comprehensively assessed in the work of Jain et al (Jain et al., 2017). The reactivity of each mAb diluted to $10 \mu \mathrm{g} / \mathrm{ml}(67 \mathrm{nM})$ towards immobilized DNP-BSA or to BSA alone was evaluated by ELISA. As can be observed on Figure $2 \mathrm{~A}$ and Supplementary Table 1, most of the therapeutic mAbs did not recognize or did weakly recognize DNP-BSA. However, a group of 25 mAbs (or ca. 22\%) bound the aromatic compound with a substantial intensity - a binding higher than the one achieved with 10 -fold higher concentration $(100 \mu \mathrm{g} / \mathrm{ml}$ or $670 \mathrm{nM})$ of pooled human $\mathrm{IgG}$ (Fig. 1B). Interestingly, among the group of mAbs binding with high intensity to DNP, only 5 molecules (or $20 \%$ ) are mAbs approved for clinical use, whereas among the group of mAbs that demonstrated binding with lower intensity the percentage of mAbs that successfully reached clinical stage is $44.8 \%(39 / 87)$.

To elucidate in further detail their binding behaviour, we selected some of mAbs showing high reactivity to DNP and tested their interactions as a function of concentration (Fig. 1C). These analyses clearly indicated that tested mAbs bound with relatively high avidity to immobilized DNP, even though more than 20-fold difference in the binding strength was observed.

In order to understand whether the binding to DNP correlates with some specific features of variable regions of mAbs, we performed correlation analyses using Spearman's non-parametric test. The intensity of binding to DNP was correlated with the number of amino acids or groups of amino acids in the CDRs and framework regions of $V_{H}$ and $V_{L}$ regions. The statistical analyses revealed that the binding of mAbs to DNP-BSA weakly but significantly correlated with a reduced number of negatively charged residues (sum of Asp and Glu) in CDR H3 (Fig. 3). This 
result might explain the observed positive correlation between the reactivity to DNP-BSA with higher value of isoelectric point of CDR H3. The binding to DNP also positively correlated with presence of the polar amino acid residue Gln in CDR H3. A negative correlation of DNP binding and numbers of lle residues in $\mathrm{CDR} \mathrm{H} 2$ was observed (Fig. 3). The DNP binding was also positively correlating with the presence of Pro in CDR $\mathrm{H} 1$ and negatively correlated with the presence of this amino acid residue in framework region 3. The reactivity to DNP showed significant correlations with different sequence traits in $V_{L}$ region and more specifically in CDR L1, CDR L2 and all framework regions (Fig. 3). Similarly as in the case of $V_{H}$, a significant correlation between DNP binding and reduced number of negatively charged amino acid residues in CDR L1 and framework region 1 was detected (Fig. 3).

Further, we selected 5 mAbs that manifest substantial reactivity to DNP-BSA and for which the crystal structures of complexes with their target antigens are available. We depicted the aromatic and charged amino acid residues displayed on the interacting molecular surfaces. These analyses reveal that both antigen-binding sites and the target epitopes are reach in charged and aromatic amino acid residues (Fig. S1). As predicted by correlation analyses, the antigen-binding sites of DNP-binding mAbs were predominantly deprived of negatively charged residues, and carry mostly positive charges, whereas the corresponding epitopes were enriched in negative charges (Fig. S1).

The frequency distribution of genes encoding $V$ regions of mAbs that showed substantial binding to DNP did not considerably differ from the genes encoding mAbs that did not bind to DNP (Fig. S2). Moreover, all gene families encoding VH and VL were used by both mAbs that show high and low capacity to binding to DNP. These observations suggest that the reactivity 
towards DNP of Abs is mediated by the unique characteristics of antigen-binding site but not by any conserved binding site for the aromatic substances in the Ig molecule.

The repertoire of mAbs used in this study overlaps with a part of the repertoire used in study of Jain and al, where the biophysical characteristics and polyreactivity of 137 mAbs were assessed by 12 different assays (Jain et al., 2017). All these assays have been used in the literature for detection of different types of developability issues. We used the data from the study of Jain to correlate the binding intensity to DNP-BSA with biophysical properties of importance for developability. We also used the data from a previous study where the reactivity of mAbs towards heme was analysed (Lecerf et al., 2021). The Spearmen's non-parametric correlation analyses revealed strong correlations of capacity of mAbs for binding to immobilized DNP with different developability parameters (Fig. 4). Thus, DNP binding positively correlated with the values from three techniques used for analysis of mAbs polyreactivity - ELISA $(\rho=0.55, P<$ 0.0001), baculovirus particles assay (BVP, $\rho=0.54, P<0.0001$ ) and polyspecificity reagent assay (PSR, $\rho=0.49, P<0.0001$ ). This result fully confirms the capacity of DNP to detect human polyreactive Abs that was reported before for polyclonal IgG samples from healthy donors or patients with autoimmune disease (Berneman et al., 1993; Bruley-Rosset et al., 2003; S. Gunti et al., 2015; S. Gunti and Notkins, 2015). mAbs reactivity to DNP also strongly correlated with increased tendency for self-association of mAbs (analysed by two independent techniques ACSINS, $\rho=0.45, P<0.0001$ and CSI, $\rho=0.44, P<0.0001$ ) as well as with increased reactivity to polyclonal human IgG (CIC or cross interaction chromatography, $\rho=0.40, P<0.005)$. Notably, increased binding to DNP correlated with lower expression yields of mAbs (HEK titer, $\rho=-0.32$, $P=0.0005)$. A weak correlation of increased binding to DNP with accelerated stability of mAbs 
(AS, $\rho=-0.20, P<0.033$ was also detected (Fig. 4). In a recent study we evaluated the interaction of mAbs with the biological cofactor molecule heme (Lecerf et al., 2021). Here, we analysed the correlation of the reactivities of mAbs to both aromatic compounds. These analyses revealed a strong positive correlation $(\rho=0.53, P<0.0001)$ between binding of mAbs to DNP with the reactivity towards heme.

\section{Discussion}

In the current study we demonstrated that a considerable number of clinical-stage mAbs bind with relatively high avidity to surface immobilized DNP-BSA. The reactivity to DNP of mAbs correlated with different developability issues - polyreactivity, tendency for self-association, decreased stability and lower expression yields in eukaryotic cells. Importantly, the binding of mAbs to DNP-BSA strongly correlated to three independent assays for assessment of polyreactivity of Abs i.e. ELISA with panel of antigens, assay with cell lysate and ELISA assay based on baculovirus. This study also revealed that the percentage of mAbs that are approved for clinical use is more than 2-fold higher in mAbs that show no or only low reactivity for DNP as compared with mAbs bindings strongly to DNP. This observation implies that assessing the reactivity to DNP has the potential to predict the likelihood of a given mAbs to reach successfully the clinical practice.

Previous studies demonstrated that all healthy humans have endogenous Abs with reactivity towards DNP (Farah, 1973; Karjalainen and Makela, 1976; Ortega et al., 1984). The origin of these Abs in human immune repertoires is not understood. Importantly, it was observed that 
DNP-binding Abs manifest antigen-binding polyreactivity (Berneman et al., 1993; Bruley-Rosset et al., 2003; Ortega et al., 1984). Our study revealed that in addition to polyclonal IgG obtained from healthy humans, the reactivity to DNP is present even in repertoire of mAbs, rationally developed as therapeutics. This observation strongly suggests that the mAbs that bind DNP are not generated as result of immune responses to xenobiotics as it was hypothesized before (McEnaney et al., 2012). Rather, it is more likely the reactivity to DNP is an intrinsic property of certain Ig molecules determined by particular physiochemical and topological features of the antigen-binding site.

DNP is an aromatic compound with low molecular weight. It has two nitro groups that endow the molecule with a polar character. The nitro groups in dinitrophenyl at neutral $\mathrm{pH}$ bear negative charge because of electron withdrawing effect of oxygen atoms. The presence of these charges can well explain the significant correlation of mAbs binding to DNP with the decreased numbers of negatively charged amino acid residues in CDR H3, CDR L1 and framework region 1 . This observation is also in line with the data reported in different studies showing that polyreactivity of Abs is often associated with more positively charged antigenbinding sites or CDR H3 (Datta-Mannan et al., 2015; Lecerf et al., 2019; Rabia et al., 2018; Raybould et al., 2019; Schoch et al., 2015; Shehata et al., 2019). The tendency for selfassociation and reactivity to other human IgG of mAbs was demonstrated to correlate strongly with antigen binding polyreactivity (Jain et al., 2017). This can explain the observation that the reactivity of mAbs to DNP also positively correlated with self-binding and cross-reactivity to other human IgG. The statistical analyses of CDR sequences and the reactivities to DNP could not, however, unambiguously clarify the observed significant correlations of DNP recognition 
and lower expression yields of mAbs. Previous studies demonstrated that the expression yields of mAbs positively correlate with protein stability (Jain et al., 2017). Indeed the reactivity to DNP also significantly correlated with lower stability as evaluated in an assay that subject Abs to stress conditions to test their stability (accelerated stability).

In a recent study we investigated the interaction of clinical-stage mAbs to heme - an ironcontaining cofactor molecule (Lecerf et al., 2021). Here we observed a statistically significant correlation in reactivities of mAbs for heme and DNP. The binding to heme correlated with intrinsic polyreactivity of mAbs and with the presence of higher number of amino acids with positive charges into the variable regions. Therefore the recognition of aromatic compounds carrying negatively charged groups (carboxyl in the case of heme) might be a strong predictor of promiscuous behaviour of mAbs. It is possible that this tendency reflects the nature of antigens recognized by polyreactive antibodies - molecules with patches of surface exposed aromatic and negative charges. Indeed, the analyses of the molecular surfaces of antigenbinding sites of mAbs that demonstrate significant reactivity to DNP-BSA and their corresponding epitopes revealed that the both interacting entities are rich in charged and aromatic amino acid residues (Fig. S1). The antigen-binding sites of the elucidated mAbs display predominantly positive charge whereas the molecular surfaces of their cognate epitopes are mostly negatively charged.

Presence of endogenous Abs with specificity for DNP in healthy humans has been exploited by numerous studies for development of innovative therapeutics for therapy of different forms of cancer, HIV-1 and influenza virus (X. Liu et al., 2020; McEnaney et al., 2012; Murelli et al., 2009; Parker et al., 2009; A. X. Zhang et al., 2010). These studies have used Ab-recruiting molecules 
with terminal DNP moiety that efficiently bridged endogenous anti-DNP Abs, available in plasma of all healthy individuals, with surface of cancer cell, virus-infected cell or viruses. Recruitment of the human anti-DNP IgGs resulted in elicitation of effector functions and ultimate destruction of the target by activation of the complement-mediated or cell-mediated cytotoxicity (X. Liu et al., 2020; McEnaney et al., 2012; Murelli et al., 2009; Parker et al., 2009; A. X. Zhang et al., 2010). Moreover, anti-DNP reactivity was used to monitor the levels of polyreactive Abs in sera of patients with systemic lupus erythematosus (S. Gunti et al., 2015). DNP-bound to chromatography matrixes was also utilized for purification of polyreactive Abs from pooled human IgG preparations obtained from healthy individuals and exploring their immune-modulation properties (Bruley-Rosset et al., 2003). Here we provide evidence for another utility of DNP - it may be used, as a probe for detection of several important unwanted properties of therapeutic mAbs i.e. polyreactivity, homophilic binding, low stability and low expression in eukaryotic cells. It is noteworthy that the proposed assay is high-throughput and requires negligible quantities of Abs. Thus, screening of for mAbs binding to DNP-BSA required dilution of IgG to concentration of $10 \mu \mathrm{g} / \mathrm{ml}$ or use of $0.5 \mu \mathrm{g}$ of $\mathrm{Ab}$ per well.

An important clue from our study for the utility of DNP ELISA as a developability assay for analysis of mAbs is the fact that the percentage of clinically approved molecules is two fold lower in group of mAbs that bind strongly to DNP as compared with the group showing not binding or binding only negligibly. Use of a simple ELISA assay with DNP-conjugated BSA may identify mAbs displaying developability issues at early stages of their development, thus guiding decisions for their feasibility or rational optimization. 


\section{Acknowledgments}

We are grateful to Dr. Max Vásquez, Dr Yingda Xu and Dr Tushar Jain (Adimab LLC, USA) for providing the monoclonal Abs and their vital support and discussions. We are grateful to $\mathrm{Dr}$ Hugo Mouquet (Institute Pasteur, Paris, France) for providing us the monoclonal antibody ED38.

\section{Funding}

This work was supported by Institut National de la Santé et de la Recherche Médicale (INSERM, France), and by a grant from "Fondation ARC pour la recherche sur le cancer", France (PJA 20171206410).

\section{References}

Avery, L.B., Wade, J., Wang, M., Tam, A., King, A., Piche-Nicholas, N., Kavosi, M.S., Penn, S., Cirelli, D., Kurz, J.C., Zhang, M., Cunningham, O., Jones, R., Fennell, B.J., McDonnell, B., Sakorafas, P., Apgar, J., Finlay, W.J., Lin, L., Bloom, L., O'Hara, D.M., 2018. Establishing in vitro in vivo correlations to screen monoclonal antibodies for physicochemical properties related to favorable human pharmacokinetics. mAbs 10, 244-255.

Berneman, A., Guilbert, B., Eschrich, S., Avrameas, S., 1993. IgG auto- and polyreactivities of normal human sera Mol. Immunol. 30, 1499-1510.

Bruley-Rosset, M., Mouthon, L., Chanseaud, Y., Dhainaut, F., Lirochon, J., Bourel, D., 2003. Polyreactive autoantibodies purified from human intravenous immunoglobulins prevent the development of experimental autoimmune diseases. Lab. Invest. 83, 1013-1023.

Datta-Mannan, A., Thangaraju, A., Leung, D., Tang, Y., Witcher, D.R., Lu, J., Wroblewski, V.J., 2015. Balancing charge in the complementarity-determining regions of humanized mAbs without affecting $\mathrm{pl}$ reduces non-specific binding and improves the pharmacokinetics. mAbs 7, 483-493. 
Dimitrov, J.D., Planchais, C., Roumenina, L.T., Vassilev, T.L., Kaveri, S.V., Lacroix-Desmazes, S., 2013. Antibody polyreactivity in health and disease: statu variabilis. J. Immunol. 191, 993-999.

Dostalek, M., Prueksaritanont, T., Kelley, R.F., 2017. Pharmacokinetic de-risking tools for selection of monoclonal antibody lead candidates mAbs 9, 756-766.

Farah, F.S., 1973. Natural antibodies specific to the 2,4-dinitrophenyl group. Immunology 25, 217-226.

Gunti, S., Kampylafka, E.I., Tzioufas, A.G., Notkins, A.L., 2015. Polyreactive antibodies in the circulation of patients with systemic lupus erythematosus Lupus 24, 1567-1569.

Gunti, S., Notkins, A.L., 2015. Polyreactive Antibodies: Function and Quantification. J. Infect. Dis. 212, S42-S46.

Hotzel, I., Theil, F.P., Bernstein, L.J., Prabhu, S., Deng, R., Quintana, L., Lutman, J., Sibia, R., Chan, P., Bumbaca, D., Fielder, P., Carter, P.J., Kelley, R.F., 2012. A strategy for risk mitigation of antibodies with fast clearance. mAbs 4, 753-760.

Jain, T., Sun, T., Durand, S., Hall, A., Houston, N.R., Nett, J.H., Sharkey, B., Bobrowicz, B., Caffry, I., Yu, Y., Cao, Y., Lynaugh, H., Brown, M., Baruah, H., Gray, L.T., Krauland, E.M., Xu, Y., Vasquez, M., Wittrup, K.D., 2017. Biophysical properties of the clinical-stage antibody landscape. Proc. Natl. Acad. Sci. U.S.A. 114, 944-949.

Jarasch, A., Koll, H., Regula, J.T., Bader, M., Papadimitriou, A., Kettenberger, H., 2015. Developability assessment during the selection of novel therapeutic antibodies. J. Pharmaceut. Sci. 104, 1885-1898.

Kaplon, H., Reichert, J.M., 2021. Antibodies to watch in 2021 mAbs 13, 1860476.

Karjalainen, K., Makela, O., 1976. Concentrations of three hapten-binding immunoglobulins in pooled normal human serum. Eur. J. Immunol. 6, 88-93.

Lecerf, M., Kanyavuz, A., Lacroix-Desmazes, S., Dimitrov, J.D., 2019. Sequence features of variable region determining physicochemical properties and polyreactivity of therapeutic antibodies. Mol. Immunol. $112,338-346$.

Lecerf, M., Kanyavuz, A., Rossini, S., Dimitrov, J.D., 2021. Interaction of clinical-stage antibodies with heme predicts their physiochemical and binding qualities Commun. Biol. 4, 391.

Liu, X., Zhang, B., Wang, Y., Haymour, H.S., Zhang, F., Xu, L.C., Srinivasarao, M., Low, P.S., 2020. A universal dual mechanism immunotherapy for the treatment of influenza virus infections Nat. Commun. 11, 5597.

Liu, Y., Caffry, I., Wu, J., Geng, S.B., Jain, T., Sun, T., Reid, F., Cao, Y., Estep, P., Yu, Y., Vasquez, M., Tessier, P.M., Xu, Y., 2014. High-throughput screening for developability during early-stage antibody discovery using self-interaction nanoparticle spectroscopy. mAbs 6, 483-492. 
McEnaney, P.J., Parker, C.G., Zhang, A.X., Spiegel, D.A., 2012. Antibody-recruiting molecules: an emerging paradigm for engaging immune function in treating human disease. ACS Chem. Biol. 7, 11391151.

Muhumuza, L., Segre, D., Segre, M., 1998. Antibodies with idiotypic and anti-idiotypic reactivity (epibodies) in conventional immune responses to dinitrophenylated carriers Immunology 93, 572-580.

Murelli, R.P., Zhang, A.X., Michel, J., Jorgensen, W.L., Spiegel, D.A., 2009. Chemical control over immune recognition: a class of antibody-recruiting small molecules that target prostate cancer. J. Am. Chem. Soc. 131, 17090-17092.

Ortega, E., Kostovetzky, M., Larralde, C., 1984. Natural DNP-binding immunoglobulins and antibody multispecificity. Mol. Immunol. 21, 883-888.

Parker, C.G., Domaoal, R.A., Anderson, K.S., Spiegel, D.A., 2009. An antibody-recruiting small molecule that targets HIV gp120. J. Am. Chem. Soc. 131, 16392-16394.

Rabia, L.A., Zhang, Y., Ludwig, S.D., Julian, M.C., Tessier, P.M., 2018. Net charge of antibody complementarity-determining regions is a key predictor of specificity. Prot. Engin. Design Select. 31, 409-418.

Raybould, M.I.J., Marks, C., Krawczyk, K., Taddese, B., Nowak, J., Lewis, A.P., Bujotzek, A., Shi, J., Deane, C.M., 2019. Five computational developability guidelines for therapeutic antibody profiling. Proc. Natl. Acad. Sci. U. S. A. 116, 4025-4030.

Schoch, A., Kettenberger, H., Mundigl, O., Winter, G., Engert, J., Heinrich, J., Emrich, T., 2015. Chargemediated influence of the antibody variable domain on FcRn-dependent pharmacokinetics. P Proc. Natl. Acad. Sci. U. S. A. 112, 5997-6002.

Sharma, V.K., Patapoff, T.W., Kabakoff, B., Pai, S., Hilario, E., Zhang, B., Li, C., Borisov, O., Kelley, R.F., Chorny, I., Zhou, J.Z., Dill, K.A., Swartz, T.E., 2014. In silico selection of therapeutic antibodies for development: viscosity, clearance, and chemical stability. Proc. Natl. Acad. Sci. U. S. A. 111, 1860118606.

Shehata, L., Maurer, D.P., Wec, A.Z., Lilov, A., Champney, E., Sun, T., Archambault, K., Burnina, I., Lynaugh, H., Zhi, X., Xu, Y., Walker, L.M., 2019. Affinity Maturation Enhances Antibody Specificity but Compromises Conformational Stability. Cell Rep. 28, 3300-3308 e3304.

Sigounas, G., Harindranath, N., Donadel, G., Notkins, A.L., 1994. Half-life of polyreactive antibodies. J. Clin. Immunol. 14, 134-140.

Steinmeyer, D.E., McCormick, E.L., 2008. The art of antibody process development. Drug Discov. Today $13,613-618$. 
Xu, Y., Roach, W., Sun, T., Jain, T., Prinz, B., Yu, T.Y., Torrey, J., Thomas, J., Bobrowicz, P., Vasquez, M., Wittrup, K.D., Krauland, E., 2013. Addressing polyspecificity of antibodies selected from an in vitro yeast presentation system: a FACS-based, high-throughput selection and analytical tool. Prot. Eng. Design Select. 26, 663-670.

Xu, Y., Wang, D., Mason, B., Rossomando, T., Li, N., Liu, D., Cheung, J.K., Xu, W., Raghava, S., Katiyar, A., Nowak, C., Xiang, T., Dong, D.D., Sun, J., Beck, A., Liu, H., 2019. Structure, heterogeneity and developability assessment of therapeutic antibodies. mAbs 11, 239-264.

Zhang, A.X., Murelli, R.P., Barinka, C., Michel, J., Cocleaza, A., Jorgensen, W.L., Lubkowski, J., Spiegel, D.A., 2010. A remote arene-binding site on prostate specific membrane antigen revealed by antibodyrecruiting small molecules. J. Am. Chem. Soc. 132, 12711-12716.

Zhang, Y., Wu , L., Gupta, P., Desai, A.A., Smith, M.D., Rabia, L.A., Ludwig, S.D., Tessier, P.M., 2020. Physicochemical Rules for Identifying Monoclonal Antibodies with Drug-like Specificity Mol. Pharm. 17, 2555-2569.

\section{Legends to figures}

Figure 1. Reactivity of human IgG to DNP-BSA. (A) Binding of human pooled IgG (IVIg) to immobilized DNP-BSA. The pooled IgG was diluted from 33.5 to $0.065 \mu \mathrm{M}$ (two-fold dilution step). Each symbol represents the average optical density (492 nm) \pm SD from three technical replicates. A representative result from two independent experiments is shown. (B) Binding of human monoclonal IgG1 Abs - ED38 (blue line and symbols) and N07G05 (red line and symbols) to immobilized DNP-BSA. The monoclonal Abs were diluted from 200 to $0.01 \mathrm{nM}$ (three-fold dilution step). Each symbol represents the average optical density (492 nm) \pm SD from three technical replicates. A representative result from two independent experiments is displayed.

Figure 2. Interaction of clinical-stage mAbs with DNP-BSA. (A) A panel depicting the reactivity of 112 IgG1 mAbs. All Abs were diluted to $10 \mu \mathrm{g} / \mathrm{ml}(67 \mathrm{nM})$ and the binding of each molecule was 
tested in triplicate. As a control binding of $100 \mu \mathrm{g} / \mathrm{ml}(670 \mathrm{nM})$ of human pooled IgG (IVIg) was assessed. First the ratio of average binding to DNP-BSA versus unconjugated carrier (BSA alone) was determined for all mAbs and for pooled IgG. Next the fold increase in the binding as compered with the one for pooled IgG was determined and shown on the plot. The reactivity of $100 \mu \mathrm{g} / \mathrm{ml}$ of pooled IgG is equal to 1 unit and it is indicated with dashed line in the plot. The red circles depict the reactivity of mAbs that are not yet approved for clinical use. The blue circles indicate the approved mAbs. (B) Interaction of selected mAbs with immobilized DNPBSA. All selected Abs except the negative control, Natalizumab, manifest reactivity higher than $100 \mu \mathrm{g} / \mathrm{ml}(670 \mathrm{nM})$ pooled IgG. The mAbs were diluted $200-0.27 \mathrm{nM}$ (three-fold dilution step). Each symbol indicates average optical density (492 $\mathrm{nm}$ ) obtained from technical duplicates.

Figure 3. Sequence features of variable regions of DNP-binding mAbs. Nonparametric Spearman's correlation analyses between the binding intensity to DNP-BSA of mAbs and V domain mutations as well as frequencies of particular amino acid residues or types of amino acid in CDRs 1-3 and framework regions 1-4 of heavy and light Ig chains. Correlation of DNP binding with hydrophobicity index (GRAVY) and isoelectric point ( $\mathrm{pl}$ ) of different part of $\mathrm{V}$ regions of mAbs are also presented. The red indicates positive correlation the blue indicates negative correlation. The value of $\rho$ is presented as intensity of colours. The statistical significance ( $P$ value) of the correlation is presented with symbols.

Figure 4. Correlation of binding of mAbs to DNP-BSA with important developability parameters used for assessment of therapeutic Abs. (A) Correlation plots from nonparametric Spearman's correlation analyses depicting the reactivity of 112 mAbs towards DNP-BSA and the measurable 
from different assays published in the study of Jain et al (Jain et al., 2017). (B) Heat-map summarizing the statistical analyses in panel A. 
Figure 1

A

B
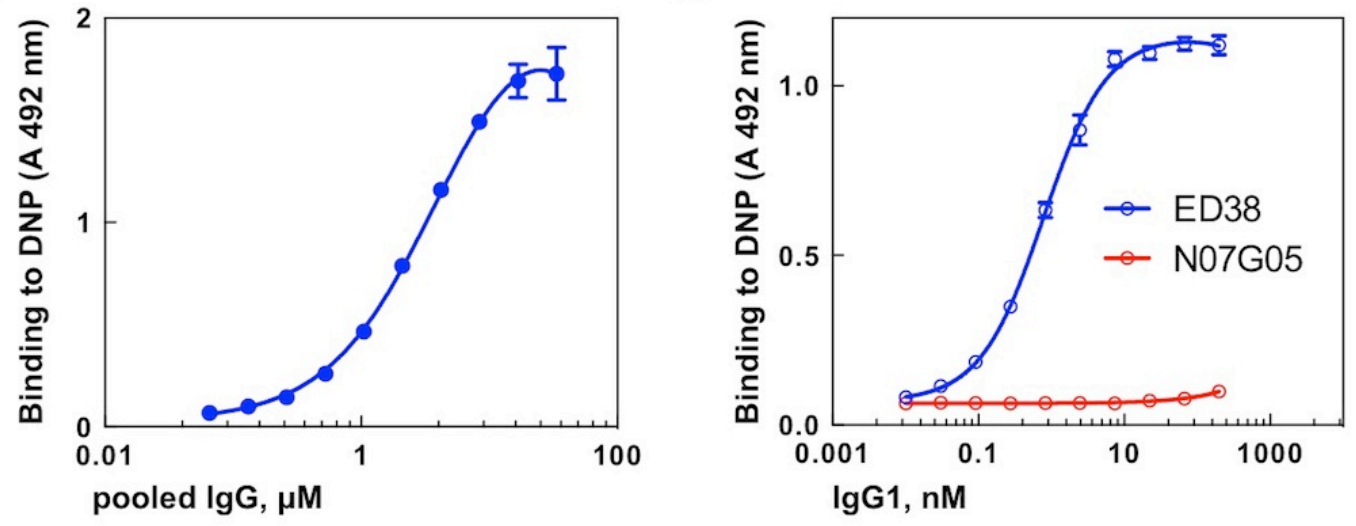
Figure 2

A

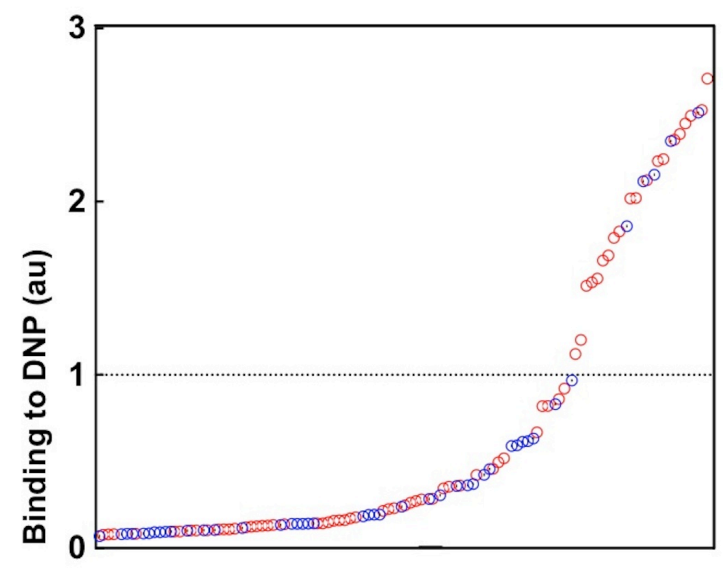

mAbs

B
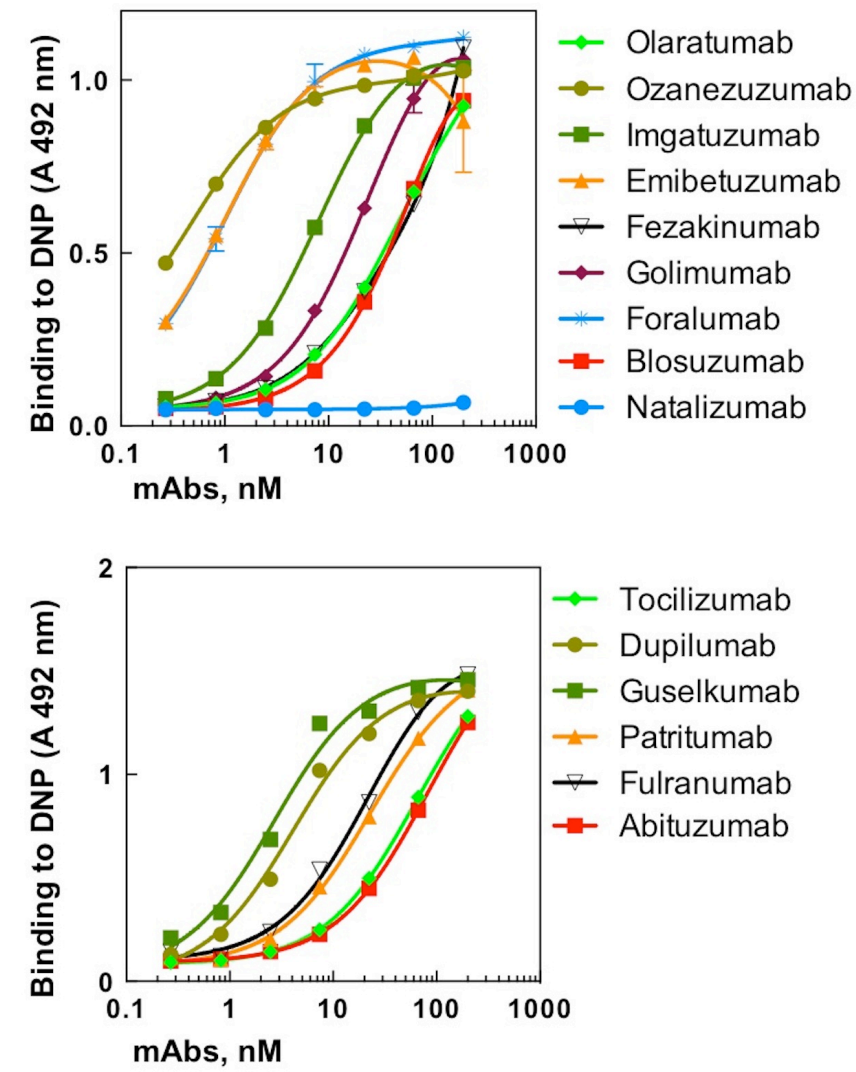
Figure 3

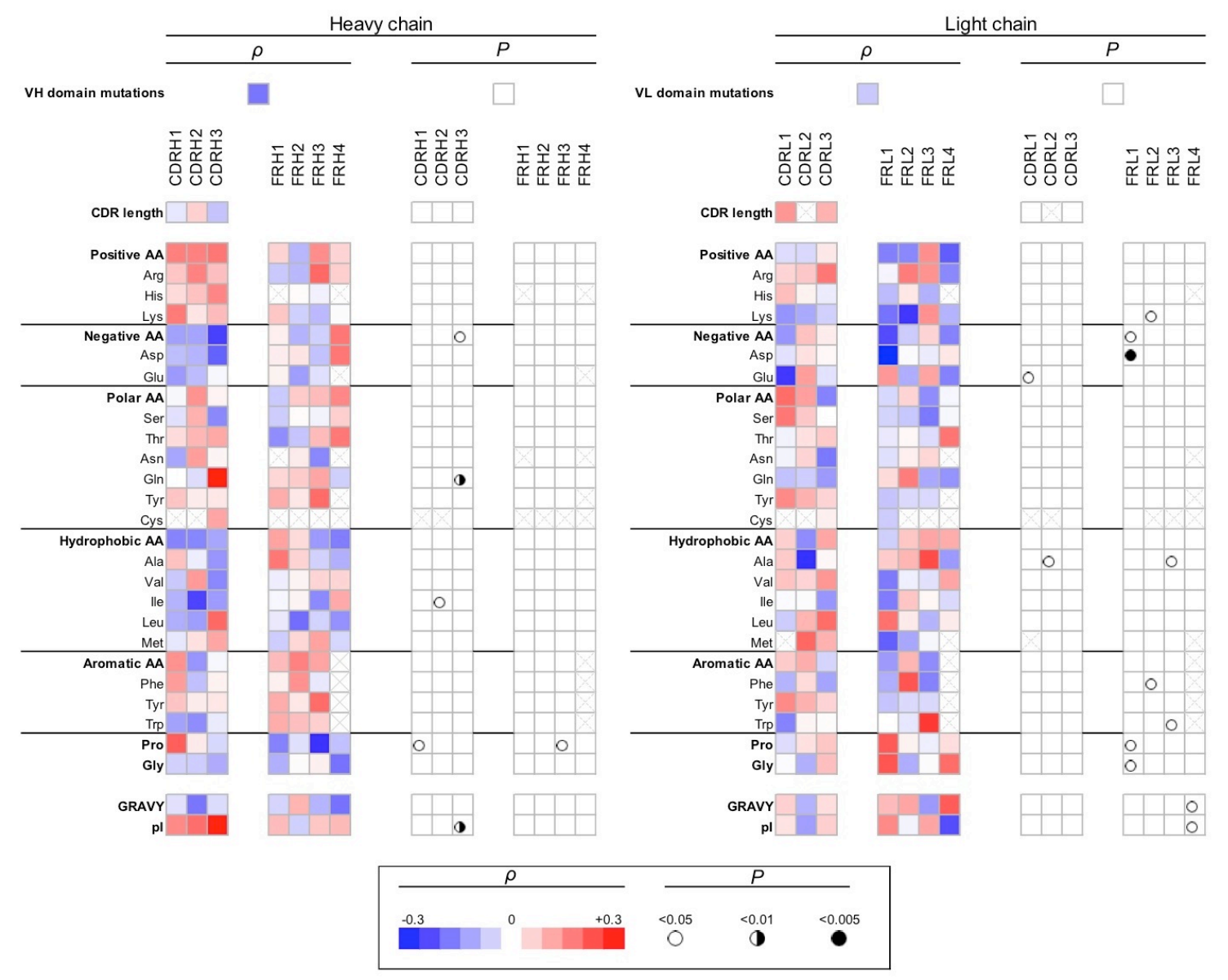


Figure 4

A
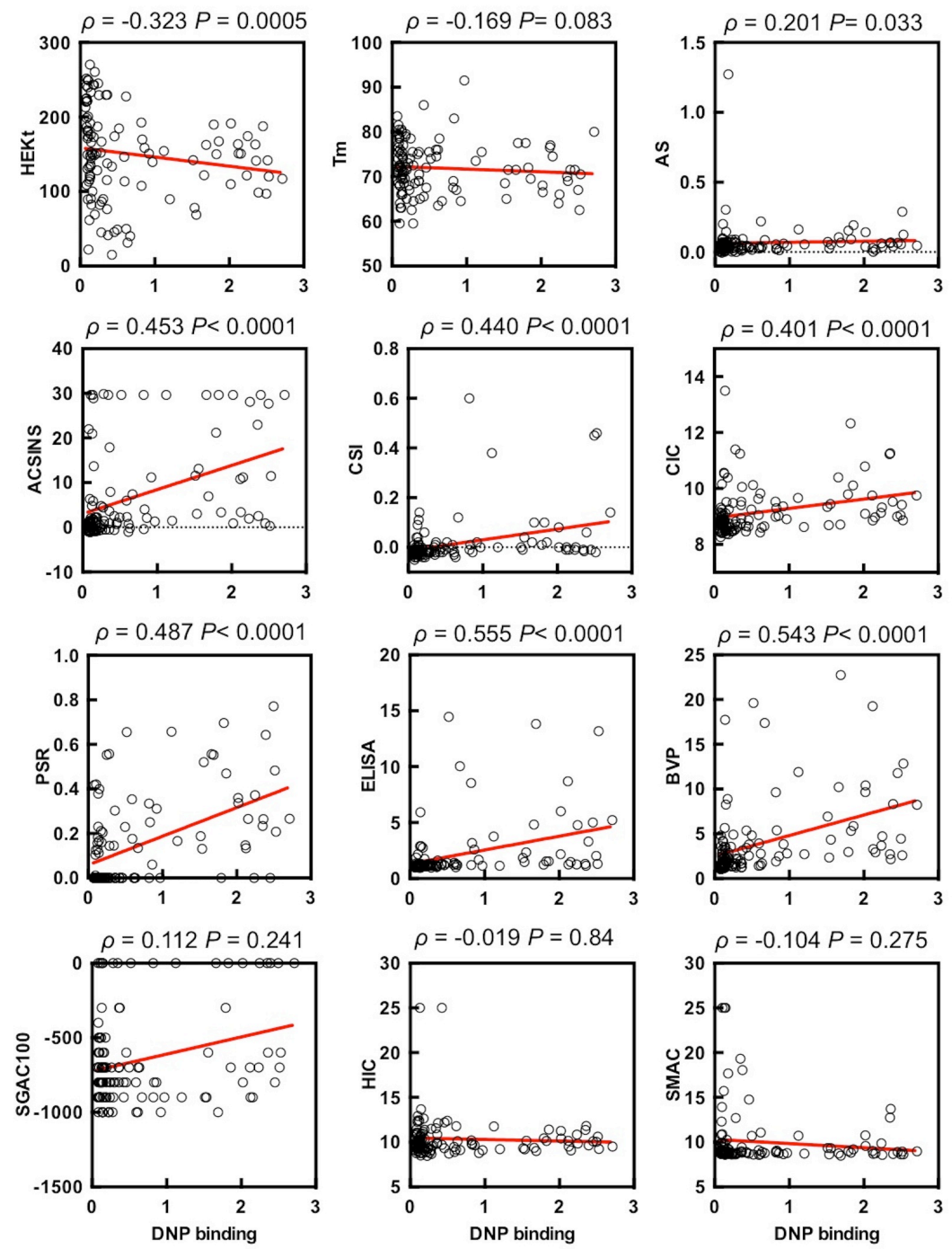

B

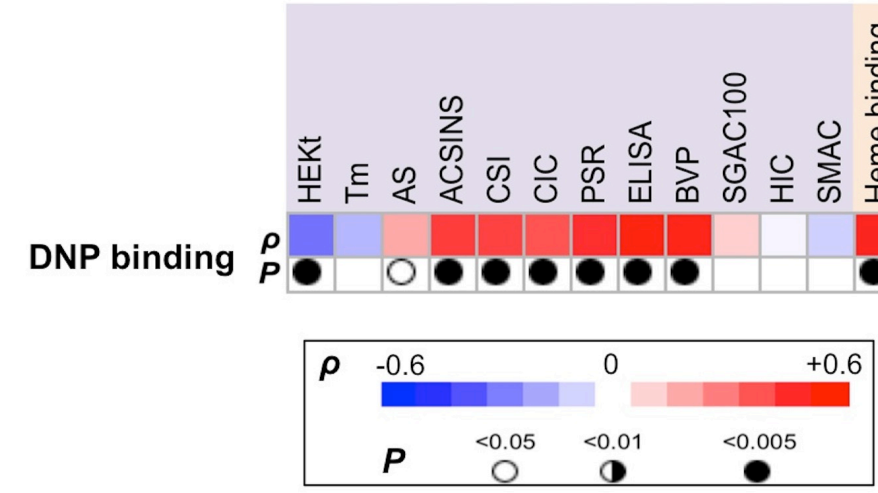

